\title{
Paradigm Shift in Metastatic Malignant Melanoma
}

\author{
Alper SEVINC \\ Gaziantep University, Faculty of Medicine, Department of Medical Oncology, Gaziantep, TURKEY
}

\begin{abstract}
Substantial advancements have been made in the treatment of metastatic malignant melanoma, for which therapeutic options were quite limited until recently, with the elucidation of molecular pathways that play role in the development and progression of the disease. Although remarkable improvement has been achieved in survival rates with these advancements, a satisfactory response rate could not be obtained or high response rates could not be maintained. In this paper, therapeutic options for metastatic melanoma, particularly ipilimumab and vemurafenib that are also in use in our country, were summarized.
\end{abstract}

Keywords: Metastatic melanoma, Ipilimumab, Vemurafenib, Paradigm

\section{ÖZET}

\section{Metastatik Malign Melanomda Paradigma Değişimi}

Yakın bir zamana kadar tedavi seçeneklerinin sınırı olduğu metastatik malign melanomda, hastalı̆̆ın gelişiminde ve progresyonunda rol oynayan moleküler yolakların ortaya konmasıyla, hastalı̆ı̆ tedavisinde önemli gelişmeler kaydedilmiştir. Bu gelişmelerle birlikte hastaların sağkalım oranlarında önemli bir iyileşme sağlanmasına karşın, yanıt oranlarında istenilen düzeye ulaşılamamakta ya da ulaşılan yüksek yanıt oranları korunamamaktadır. Bu yazıda, ülkemizde de kullanımda olan ipilimumab ve vemurafenib başta olmak üzere metastatik melanom tedavileri özetlenmiştir.

Anahtar Kelimeler: Metastatik melanom, Ipilimumab, Vemurafenib, Paradigma 


\section{INTRODUCTION}

Melanoma is an important healthcare problem that has become one of the most common cancer types with a dramatic increase in incidence over the last decades. ${ }^{1}$ Although the skin is the most common site of origin, noncutaneous melanocytes such as those lining the choroidal layer of the eye, mucosal surfaces of the respiratory, gastrointestinal, and genitourinary tracts, and the meninges may also undergo malignant transformation. ${ }^{2}$

Metastatic melanoma is the most aggressive form of skin cancer with approximately 13,000 deaths per year and a median overall survival (OS) ranging between 8 and 18 months. ${ }^{3}$ Until recently, therapeutic options for metastatic melanoma were limited; the only approved therapeutic options were dacarbazine, an alkylating agent with a very limited response rate and a median OS of 8 months, and interleukin 2 (IL2), an immunomodulatory agent with an even lower response rate and severe toxicity. ${ }^{3}$ With these agents, remissions are infrequent and usually of short duration. Moreover, the treatment is primarily palliative as their effect on survival is not clear. ${ }^{1,4,5}$

In 2011, there has been a paradigm shift in the treatment of metastatic melanoma with the introduction of ipilimumab (Yervoy ${ }^{\mathrm{TM}}$, Bristol-Myers Squibb, Princeton, NJ) and vemurafenib (Zelboraf, Plexxikon/Roche, Auckland, NZ). These agents have both been shown to prolong survival in advanced stage melanoma patients as compared to standard treatments. The clinical benefits of these agents in melanoma have been realized after years of research in the molecular pathogenesis of the disease. With a history of multiple negative phase III studies, melanoma is now a tumor type for the clinical evaluation of paradigm-shifting therapeutic strategies.

\section{RECENT ADVANCES}

\subsection{Immunotherapy}

\subsubsection{Ipilimumab}

Melanoma has long been recognized as an immunogenic tumor due to the presence of infiltrating lymphocytes in resected melanoma, occasional spontaneous regressions, and clinical responses to immune stimulation. ${ }^{3}$ Advancements in the unders- tanding of immune cellular signaling pathway abnormalities that promote the development and progression of melanoma has led investigators to search for novel strategies to overcome immune evasion. ${ }^{3}$ Cytotoxic T-lymphocyte-associated antigen 4 (CTLA-4) is a immunomodulatory molecule expressed on activated T-cells and T-regulatory cells. ${ }^{6}$ This molecule delivers a negative costimulatory signal that downregulates T-cell activity. Ipilimumab is a fully human IgG1 monoclonal antibody that blocks this molecule, and thus enhances T-cell activity and recognition of the tumor by T-cells not only in malignant melanoma but also in several cancer types. Ipilimumab is now the first treatment in a randomized study to demonstrate a clear OS benefit in metastatic melanoma. ${ }^{6}$

In a randomized, double-blind phase II study, previously treated, advanced stage melanoma patients were randomized to receive a fixed dose of ipilimu$\mathrm{mab}$ of either of $0.3 \mathrm{mg} / \mathrm{kg}, 3 \mathrm{mg} / \mathrm{kg}$, and $10 \mathrm{mg} / \mathrm{kg}$ every 3 weeks for four cycles followed by maintenance therapy every 3 months. It was observed that the best overall response rate was increased in a dose-dependent fashion, and ipilimumab showed clinical activity at doses of $3 \mathrm{mg} / \mathrm{kg}$ and higher., ${ }^{5,6} \mathrm{In}$ another phase II study, chemotherapy-naïve metastatic melanoma patients were randomized to receive ipilimumab at $3 \mathrm{mg} / \mathrm{kg}$ every 4 weeks for four cycles either alone or in combination with dacarbazine $250 \mathrm{mg} / \mathrm{m}^{2} /$ day for 5 days for up to six courses. The objective response rates were $5.4 \%$ and $14.3 \%$ in the monotherapy and combination therapy arms, respectively, and there was no significant difference between the two arms in this respect. ${ }^{67}$ Further studies of ipilimumab at a dose of $10 \mathrm{mg} / \mathrm{kg}$ showed best overall response rates ranging between $5.8 \%$ and $15.8 \%{ }^{6}$

In a phase III study, patients with unresectable, advanced stage melanoma that had progressed while the patients were receiving therapy were randomized to receive ipilimumab plus glycoprotein 100 (gp100) peptide vaccine, ipilimumab alone, or gp100 alone. All treatments were administered every 3 weeks for four cycles. The primary efficacy endpoint of the study was OS, which was shown to be prolonged in ipilimumab monotherapy and combination arms as compared to gp100 monotherapy arm (10 months and 10.1 months vs. 6.4 months, 


\begin{tabular}{|lll|}
\hline Table 1. Comparison of CTLA-4 blocker, ipilimumab and BRAF inhibitor, vemurafenib in phase III studies \\
\hline & Ipilimumab & Vemurafenib \\
\hline Indication & Any melanoma & $\begin{array}{l}\text { Unresectable or metastatic melanoma } \\
\text { harboring a BRAF V600E mutation } \\
\text { Inhibition of mutant BRAF V600E }\end{array}$ \\
Mechanism of action & Blockade of CTLA-4 & Oral \\
Route of administration & Intravenous & Wild-type BRAF \\
Contraindication & Autoimmune diseases & $48 \%$ \\
Best overall response rate & $15.2 \%$ & 5.3 months \\
PFS & 2.86 months & No \\
Durable response & Yes & Immune-mediated AEs most commonly \\
Adverse events & affecting the skin and gastrointestinal tract & and fatigue events including SCC, arthralgia \\
& & \\
\hline
\end{tabular}

respectively). Moreover, there was no additional benefit of the vaccine as there was no significant difference between the two ipilimumab arms with respect to OS.8 On the basis of these results, ipilimumab received United States Food and Drug Administration (FDA) approval for the treatment of metastatic melanoma in 2011. ${ }^{6}$ Following the approval of ipilimumab by the FDA, the drug was integrated into the National Comprehensive Cancer Network (NCCN) melanoma guidelines. More recently, a footnote regarding re-induction with ipilimumab was added to the section in the guidelines on metastatic disease. Re-induction with ipilimumab was emphasized in patients not experiencing a significant toxicity during previous therapy and relapse after clinical response or progress after stable disease $>3$ months. In the above-mentioned phase III study randomizing previously treated patients with metastatic melanoma to receive ipilimumab plus gp100, ipilimumab alone, or gp100 alone, the disease control rate was found 52\%-67\% among those receiving re-induction with ipilimumab, and the safety profile during re-induction was consistent with that of overall study. ${ }^{9}$

Another phase III study randomized previously untreated metastatic melanoma patients to receive dacarbazine in combination with ipilimumab or placebo. It was observed that OS was significantly longer in patients receiving ipilimumab plus dacarbazine as compared to those receiving placebo plus dacarbazine (11.2 months vs. 9.1 months). The sur- vival benefit was also observed when the patients were grouped according to age, sex, Eastern Cooperative Oncology Group (ECOG) performance status, baseline serum lactate dehydrogenase level, and substage of the disease. ${ }^{10}$ However, response rates were not satisfactory in both phase III studies (Table 1). ${ }^{3}$ The low clinical response rate with ipilimumab treatment has been suggested to be increased by either through improved patient selection, through combination with other therapies or by applying higher doses. ${ }^{3}$ In an attempt to increase response rates and improve clinical outcomes, two drugs may be used sequentially; a BRAF inhibitor to reduce the tumor load and then use ipilimumab to maintain the response. ${ }^{11}$ The efficacy of this approach was tested in a retrospective analysis of patients receiving vemurafenib or dabrafenib and ipilimumab as part of a trial or expanded access program. In this analysis, 12 out of 28 patients receiving a BRAF inhibitor first were unable to complete treatment with ipilimumab due to rapid disease progression. A logistic regression showed elevated LDH, an ECOG performance status of 1 and the presence of brain metastases to be the most significant risk factors for rapid progression. It was suggested that patients having two or more of these risk factors could potentially benefit from ipilimumab as the first part of the sequential treatment regimen. Of note, there was no correlation between the number of risk factors at baseline and rate of disease progression in patients who received ipilimumab first and were subsequently treated with a 
BRAF inhibitor upon disease progression. ${ }^{11}$ Furthermore, my colleagues and I reported a case of malignant melanoma with multiple brain metastases that survived for 40 months with the sequential use of ipilimumab and vemurafenib. ${ }^{12}$

Currently, there is no reliable predictor of benefit for ipilimumab. ${ }^{3}$ One group reported that the presence of a BRAF mutation does not predict clinical benefit of ipilimumab. ${ }^{3}$ On the other hand, it has been shown that ipilimumab increases the frequency of T-cells with inducible co-stimulatory molecule (ICOS) and that ICOS T-cells are necessary for response to ipilimumab. ${ }^{3}$ In order to draw a definite conclusion about this issue, it has to be determined whether or not baseline ICOS T-cells predict benefit of ipilimumab. ${ }^{3}$ And, there is evidence suggesting that a higher response rate can be achieved when ipilimumab is administered at a higher dose $(10 \mathrm{mg} / \mathrm{kg})$ than the dose approved by the FDA. ${ }^{3}$

In addition to better patient selection, ipilimumab has been combined with other modalities to increase response rates. Although the phase III study of ipilimumab plus dacarbazine failed to show an improved disease control rate and overall response rate with ipilimumab therapy, this question was not addressed in that particular study. ${ }^{10}$ In the randomized phase II study of ipilimumab with or without dacarbazine, patients receiving ipilimumab plus dacarbazine had higher disease control and best overall response rates, although the difference between the two treatment arms did not reach statistical significance. ${ }^{7}$ In a phase II study of ipilimumab and temozolamide, an overall disease control rate of $67 \%$ was achieved, which is much higher than seen in single agent studies. ${ }^{13}$ In a phase I study of ipilimumab and bevacizumab, response was achieved in 8 , disease control was achieved in 14 out of 21 patients with unresectable, advanced-stage melanoma; however, immune-related toxicity also seemed to be enhanced with this combination. ${ }^{14}$

\subsubsection{Anti-PD-1}

Programmed death-1 (PD-1), as CLTA-4, is a costimulatory molecule for T-cell activation and has become a target for cancer immunotherapy on the basis of experimental studies suggesting that the blockage of the interaction between PD-1 and its ligand PD-L1 potentiates immune response and mediates antitumor immunity. ${ }^{15,16}$ Indeed, early phase studies have shown that agents targeting the interaction between these two molecules exert antitumor activity and induce durable tumor response in advanced cancers, including melanoma. ${ }^{15,16}$

\subsection{Targeted Treatment}

\subsubsection{BRAF Inhibitors}

\subsubsection{Vemurafenib}

In 2002, it was discovered that cutaneous melanoma is a molecularly heterogeneous disease harboring an activating mutation in the gene encoding for the serine-threonine protein kinase B-raf (BRAF) in two thirds of patients. The vast majority of the activating BRAF mutations involve a valine for glutamate substitution at codon 600 (V600E), which results in constitutive activation of the mitogen-activated protein kinase pathway (MAPK), leading to oncogenic cell proliferation. ${ }^{3,17}$ Recently, highly selective BRAF inhibitors that are capable of silencing BRAF V600E mutations with little effect on wild-type BRAF have been developed. Vemurafenib (PLX-4032, RG7204) is a specific inhibitor of mutant BRAF kinase, and is the second agent, after ipilimumab, that has been shown to improve OS in patients with advanced stage melanoma. $^{1,3}$

In a phase I study, vemurafenib induced partial or complete response in $81 \%$ of patients with metastatic melanoma harboring a BRAF V600E mutation. ${ }^{18}$ In a pivotal phase II study of vemurafenib, overall response was achieved in $52.3 \%$ of previously treated patients with BRAF V600E mutation-positive metastatic melanoma. ${ }^{19}$ These results were collaborated with a more recent phase II study, in which vemurafenib induced clinical response in $53 \%$ of patients with previously treated BRAF V600E mutation-positive metastatic melanoma. ${ }^{20} \mathrm{~A}$ phase III randomized study comparing vemurafenib and dacarbazine showed that vemurafenib treatment significantly prolonged 6-month OS as compared to dacarbazine ( $84 \%$ vs. 64\%). The interim analysis for OS and final analysis for progression-free survival (PFS) demonstrated a relative reduction of $63 \%$ in the risk of death and of $74 \%$ in the risk of either death or disease progression with vemurafenib when compared to dacarbazine. The most common adverse events observed during treatment with ve- 
murafenib were cutaneous events, arthralgia and fatigue (Table 1). Cutaneous squamous-cell carcinoma (SSC), keratoacanthoma, or both developed in $18 \%$ of patients which were treated through simple excision. ${ }^{21}$ This phase III study was the first-in-melanoma showing an inhibition of an aberrantly overactive signaling pathway improving survival. Based on these results, the FDA approved vemurafenib in 2011. ${ }^{1}$

\subsubsection{Dabrafenib}

There are several other BRAF inhibitors that are currently undergoing clinical testing. Another BRAF inhibitor, dabrafenib (GSK2118436), also showed clinical activity with minimal toxicity in a phase I/II study. ${ }^{22}$ In a phase III study of patients with previously untreated BRAF V600E mutationpositive melanoma, dabrafenib showed a significant improvement in progression-free survival as compared to dacarbazine (5.1 months vs. 2.7 months). Among patients receiving dabrafenib, $50 \%$ achieved an objective response confirmed by an independent review committee..$^{23}$

Besides their efficacy in BRAF V600E mutations, vemurafenib and dabrafenib have both shown activity in V600K mutant melanomas, which is the most common non-V600E mutation in melanoma patients. ${ }^{3}$ However, these agents are not currently approved for this indication. ${ }^{3}$

In brief, both agents have shown clinical activity with high response and minimal toxicity in patients with BRAF V600E mutations; however, both therapies have a relatively short duration. 3 The most important AEs related to the use of BRAF inhibitors are cutaneous events, such SCCs and keratoacanthomas develop as a result of the paradoxical transactivation of MAPK by these agents. ${ }^{3}$

\subsubsection{Other Targeted Agents}

In addition to BRAF, molecular alterations involving oncogenes including NRAS, MEK, ERK, KIT, CDK4, CCND1, ERBB4, AKT, NEDD9, GNAQ and GNA1, transcription factors including MITF, MYC and ETV1, and tumor suppressors including CDKN2A, TP53, BAP1 and PTEN have been identified in melanoma patients. ${ }^{3,17}$ Among those, MEK is an attractive target as it is downstream of both ac- tivated BRAF and NRAS. ${ }^{1}$ It was shown in preclinical studies that the sensitivity to MEK inhibition was higher in melanoma cells harboring a BRAF mutation than those harboring activating NRAS mutations and wild-type BRAF genes. While small-molecule MEK inhibitors completely abrogated tumor growth in BRAF mutant xenografts, they showed a partial inhibitory effect on RAS mutant tumors. ${ }^{1}$ MEK inhibitors under clinical development include CI-1040, PD-0325901, trametinib (GSK1120212), and AZD6244. Early-phase studies have shown clinical activity of trametinib,24 and trametinib improved PFS and OS in patients with BRAF-mutant melanoma as compared to chemotherapeutic agents, dacarbazine and paclitaxel, in a phase III study. ${ }^{25}$ On the other hand, some pharmacological and toxicity issues limit the activity of CI1040 and PD-0325901.

Another molecular pathway involved in the pathogenesis of melanoma is c-KIT signaling pathway which plays a key role in the differentiation, proliferation, and migration of normal melanocytes. However, this tyrosine kinase receptor also contributes to the pathogenesis of a subset of melanomas that do not harbor NRAS or BRAF mutations. c-KIT mutations have been identified in a substantial proportion of melanomas originating from mucosa, acral and chronic sun-induced damaged skin. ${ }^{126,27}$

\subsection{Combined Targeted Therapies}

To overcome low response rates with ipilimumab therapy and short durations of response in targeted therapies, novel combinations of immune targeted therapies are being tested. It has been suggested that combining immunotherapy with a BRAF-targeted therapy may allow achieving high response rates and durable response rates. ${ }^{28}$ Based on preclinical studies suggesting a strong rationale for combining a BRAF inhibitor with an immune-stimulating agent, a phase I/II study was planned to assess the safety and efficacy of ipilimumab and vemurafenib combination and to show preliminary evidence of anti-tumor efficacy and survival in comparison to historical results following treatment with either agent alone in metastatic melanoma patients with V600 BRAF mutations. Unfortunately, the results from this study will not be available until 2015.11 
Another promising strategy is the BRAF/MEK inhibitor combination showing greater activity against cancer cells harboring BRAF mutations as compared to either drug alone, and decreased incidence of hyper-proliferative skin lesions related to BRAF inhibitor use. ${ }^{1}$ In general, these strategies have focused on either inhibiting additional targets within the same pathway or inhibiting a different pathway or cellular process involved in the pathogenesis or drug resistance of melanoma. ${ }^{1}$ Studies combining CTLA-4 and other immunomodulatory antibodies, and/or vaccines are also underway. These strategies are based on the current understanding of molecular or signaling pathways involving in the pathogenesis of the disease, availability of agents and although limited, preclinical data suggesting an additional benefit of combination therapy without a further increase in normal tissue toxicity. ${ }^{1}$

\subsection{Local Studies in Turkey}

To the best of our knowledge, the number of studies conducted on metastatic melanoma patients receiving ipilimumab or vemurafenib in our country is quite limited. In a retrospective analysis of 75 metastatic melanoma patients treated under an expanded access program in Turkey, it was found that none of the patients achieved a complete response with ipilimumab therapy. The disease control rate was found $36.1 \%$, and the time to progression was 2.7 months. ${ }^{29}$ Another study of ipilimumab retrospectively evaluated demographic and clinical characteristics of 20 malignant melanoma patients and reported a median PFS of 2.7 months and an OS of 8.6 months. Four of 13 patients who were screened for BRAF V600E mutations received vemurafenib after ipilimumab. Comparison of the patients who did or did not receive sequential vemurafenib therapy reveled an improved OS in those receiving sequential vemurafenib (6.3 months vs. 19 months).$^{30}$ In another retrospective study, clinical and demographic characteristics of 13 stage IV metastatic melanoma patients receiving vemurafenib were evaluated. Of the patients, 6 had multiple brain metastases, and 4 had previous ipilimumab therapy. Despite the presence of poor prognostic factors, $15.3 \%$ of the patients achieved complete response while $23.1 \%$ achieved partial response. Although the median PFS and OS were 3.45 months and 5.49 months, respectively, among the overall study population, subgroup analyses revealed median PFS of 3.30 months and a median OS of 4.54 months among patients with previous ipilimumab therapy. The comparison of patients with and without brain metastases showed a poorer PFS and OS among those with brain metastases. ${ }^{31}$

Another study from Turkey was report of a metastatic melanoma case with multiple unresectable brain metastases who survived 40 months with the sequential use of ipilimumab and vemurafenib. ${ }^{12}$

\section{CONCLUSION}

Improvements in our understanding of the molecular and signaling pathways involved in the development and progression of the disease have led to the development of targeted therapies capable of improving survival in advanced stage melanoma patients. Currently, the paradigm-shifting molecules, ipilimumab and vemurafenib are the first-line treatment options for these patients. Sequential usage of these agents may also be the leading way of treating melanoma patients in the near future. However, intrinsic and acquired resistance to these agents will allow investigators to develop novel combination strategies that will hopefully improve clinical benefit in melanoma patients.

\section{REFERENCES}

1. Monzon JG, Dancey J. Targeted agents for the treatment of metastatic melanoma. Onco Targets Ther 5: 31-46, 2012.

2. Tsao H, Chin L, Garraway LA, Fisher DE. Melanoma: from mutations to medicine. Genes Dev 26: 11311155, 2012.

3. Finn L, Markovic SN, Joseph RW. Therapy for metastatic melanoma: the past, present, and future. BMC Med 10: 23, 2012.

4. Garbe C, Eigentler TK, Keilholz U, et al. Systematic review of medical treatment in melanoma: current status and future prospects. Oncologist 16: 5-24, 2011.

5. Wolchok JD, Neyns B, Linette G, et al. Ipilimumab monotherapy in patients with pretreated advanced melanoma: a randomised, double-blind, multicentre, phase 2, dose-ranging study. Lancet Oncol 11: 155-164, 2010.

6. Patel SP, Woodman SE. Profile of ipilimumab and its role in the treatment of metastatic melanoma. Drug Des Devel Ther 5: 489-495, 2011. 
7. Hersh EM, O'Day SJ, Powderly J, et al. A phase II multicenter study of ipilimumab with or without dacarbazine in chemotherapy-naïve patients with advanced melanoma. Invest New Drugs 29: 489-498, 2011.

8. Hodi FS, O'Day SJ, McDermott DF, et al. Improved survival with ipilimumab in patients with metastatic melanoma. N Engl J Med 363: 711-723, 2010.

9. Robert C, Hodi FS, O'Day SJ, et al. Re-induction with ipilimumab, gp100 peptide vaccine, or a combination of both in a phase III study of previously-treated patients with advanced melanoma: update of clinical characteristics of patients. Ann Oncol 21: 1330, 2010.

10. Robert C, Thomas L, Bondarenko I, et al. Ipilimumab plus dacarbazine for previously untreated metastatic melanoma. N Engl J Med 364: 2517-2526, 2011.

11. Ascierto PA, Simeone E, Giannarelli D, et al. Sequencing of BRAF inhibitors and ipilimumab in patients with metastatic melanoma: a possible algorithm for clinical use. J Transl Med 10: 107, 2012.

12. Balakan $\mathrm{O}$, Süner $\mathrm{A}$, Yiğiter $\mathrm{R}$, et al. Long-term survival in metastatic malignant melanoma: ipilimumab followed by vemurafenib in a patient with brain metastasis. Intern Med 51: 2819-2823, 2012.

13. Patel SP, Bedikian AY, Papadopoulos NE, et al. Ipilimumab plus temozolomide in metastatic melanoma. $J$ Clin Oncol 29 (suppl): abstr 8579, 2011.

14. Hodi FS, Friedlander PA, Atkins MB, et al. A phase I trial of ipilimumab plus bevacizumab in patients with unresectable stage III or stage IV melanoma. J Clin Oncol 29 (suppl): abstr 8511, 2011.

15. Brahmer JR, Tykodi SS, Chow LQ, et al. Safety and activity of anti-PD-L1 antibody in patients with advanced cancer. N Engl J Med 366: 2455-2465, 2012.

16. Brahmer JR, Drake CG, Wollner I, et al. Phase I study of single-agent anti-programmed death-1 (MDX-1106) in refractory solid tumors: safety, clinical activity, pharmacodynamics, and immunologic correlates. J Clin Oncol 28: 3167-3175, 2010.

17. Jang $S$, Atkins $M B$. Which drug, and when, for patients with BRAF-mutant melanoma? Lancet Oncol 14: e60-e69, 2013.

18. Flaherty KT, Puzanov I, Kim KB, et al. Inhibition of mutated, activated BRAF in metastatic melanoma. $\mathrm{N}$ Engl J Med 363: 809-819, 2010.

19. Ribas A, Kim KB, Schuchter LM, et al. BRIM-2: An open-label, multicenter phase II study of vemurafenib in previously treated patients with BRAF V600E mutation-positive metastatic melanoma. J Clin Oncol 29 (suppl): abstr 8509, 2011.

20. Sosman JA, Kim KB, Schuchter L, et al. Survival in BRAF V600-mutant advanced melanoma treated with vemurafenib. N Engl J Med 366: 707-714, 2012.

21. Chapman PB, Hauschild A, Robert C, et al. Improved survival with vemurafenib in melanoma with BRAF V600E mutation. N Engl J Med 364: 2507-2516, 2011.
22. Kefford R, Arkenau H, Brown MP, et al. Phase $\mathrm{I} / \mathrm{ll}$ study of GSK2118436, a selective inhibitor of oncogenic mutant BRAF kinase, in patients with metastatic melanoma and other solid tumors. J Clin Oncol 28 (suppl): abstr 8503, 2010.

23. Hauschild A, Grob JJ, Demidov LV, et al. Dabrafenib in BRAF-mutated metastatic melanoma: a multicentre, open-label, phase 3 randomised controlled trial. Lancet 380: 358-365, 2012.

24. Salama AK, Kim KB. Trametinib (GSK1120212) in the treatment of melanoma. Expert Opin Pharmacother 2013 Feb 23. doi:10.1517/14656566.2013.770475

25. Flaherty $\mathrm{KT}$, Robert C, Hersey P, et al. Improved survival with MEK inhibition in BRAF-mutated melanoma. N Engl J Med 367: 107-114, 2012.

26. Minor DR, Kashani-Sabet M, Garrido M, et al. Sunitinib therapy for melanoma patients with KIT mutations. Clin Cancer Res 18: 1457-1463, 2012.

27. Ashida A, Takata M, Murata H, et al. Pathological activation of KIT in metastatic tumors of acral and mucosal melanomas. Int J Cancer 124: 862-868, 2009.

28. Ribas A. Combination therapies building on the efficacy of CTLA4 and BRAF inhibitors for metastatic melanoma. In: 48th Annual Meeting of the American Society of Oncology Educational Book. Ramaswamy Govindan (ed). June 1-5, 2012, Chicago, Illinois, 2012: 675-678.

29. Sevinc A, Turna H, Ozdogan M, et al. Multicentric ipilimumab experience in Turkish patients with metastatic melanoma: MIPI-TURK. J Clin Oncol 30(suppl): abstr e19024, 2012.

30. Balakan O, Ciltas A, Sevinc A, et al. Retrospective analysis of malignant melanoma patients receiving ipilimumab therapy. 9th National Medical Oncology Congress, Turkish Republic of Northern Cyprus, September 12-16, 2012: P91.

31. Sevinc A, Suner A, Ciltas A, et al. Retrospective analysis of malignant melanoma patients receiving vemurafenib therapy. 9th National Medical Oncology Congress, Turkish Republic of Northern Cyprus, September 12-16, 2012: P131.

\section{Correspondence:}

Prof. Dr. Alper SEVINC

Gaziantep Üniversitesi Tıp Fakültesi

Tıbbi Onkoloji bilim Dalı

GAZIANTEP / TURKEY
Tel: $\quad$ (+90.555) 2414687
Faks: (+90.342) 4720718
e-mail: sevinc@gantep.edu.tr 